\title{
Study of Countermeasure Systems for Leaks of Onscreen Content by Digital Video/Camera Shooting
}

\author{
Masaki Fujikawa and Ryosuke Kamai \\ Sohgo Security Services Co., Ltd.(ALSOK) \\ Shingo Fuchi and Yoshikazu Takeda \\ Nagoya University
}

\author{
Fumihiko Oda and Kengo Moriyasu \\ Ushio Inc.
}

\author{
Hikaru Mori and Kenji Terada \\ Tokushima University
}

\begin{abstract}
We have been engaged in the study of countermeasures to prevent the leak of onscreen content (texts, images and movies) using a digital video camera. One of the studies is the development of a transparent sheet that emits infrared (IR) light, which is applied to the screen and generates optical noise in the onscreen photographic images when recorded. Another study is the development of a method to detect video recording/camera shooting near the display as a countermeasure to prevent video recording using the IR-cut filter. This report is prepared as an advance report because it was confirmed that the feasibility of such countermeasures is enhanced.
\end{abstract}

\section{Introduction}

With expanded use of cloud computing and enhanced functionality of the digital camera (large memory size, standard movie shooting function, and increased operability), sharing of the content (images and movies) taken by the digital camera with family members or friends and posting such content on video sites open to the general public are becoming popular among the people. Today, a wide variety of content is shared and open to the public and people are enjoining such content as a new style of entertainment.

On the other hand, cases have been reported where content that should be treated as proprietary and confidential have been posted on movie sites open to the public [1], [2]. Because the LAN connected to the Internet or storage media like CDs, DVDs, and smart media are usually used for content leaks, countermeasures to prevent leakage, such as the introduction of a device that blocks the uploading of files using a specific protocol (ftp, http, etc.) or introduction of software that blocks the copying of content onto storage media are employed on PCs used in government and enterprise offices.

The authors have been engaged in the study of content leaks in the area where no effective countermeasures were proposed (i.e., shooting/recording of the onscreen contents on the PC display by the digital camera and taking such contents to outside) [3], and a case of the content leak in such fashion was actually reported, which indicates the importance of such countermeasures [4]. In this case, an air traffic controller brought his digital camera to the control room and photo shot the confidential information on the flight plans of President Obama's Air Force One and the US Air Force's unmanned aerial reconnaissance plane Global Hawk displayed on the screens, and posted such images on his blog.

The authors believe that, in order to counteract the above mentioned content leaks, countermeasures in two areas are required. One is development of a transparent sheet that emits IR light, which is attached to the screen and generates optical noise in onscreen photographic images when recorded (hereinafter called Countermeasure 1). Another one is development of the system that detects shooting of the screen in order to cope with shooting/recording using a IR cut-off filter, which nullifies the effect of the Countermeasure 1 (i.e., inclusion of the optical noise can be evaded. Hereinafter, we called Countermeasure 2). In this report, high feasibility of such countermeasures is indicated based on the descriptions about the detail of these countermeasures and the results of the experiments.

\section{Development target setting}

First, the targets to be attained by respective countermeasures were determined based on several considerations.

\subsection{Consideration 1}

Infringement of copyright where a movie projected on the screen in the theater is recorded using a video camera, and the recorded data are duplicated on DVD or posted on a video site is becoming a problem [5]. As a countermeasure to prevent video recording by the audience, Yamada et al. proposed the projection of multiple beams of IR light from behind the screen to generate optical noise in the digital camera recording (degradation of the image) [6]. According to their report, the wavelength of IR light that is easily detected by the CCD and CMOS image sensors but not detected as visible 
light by the human eye (i.e., IR light that does not impair the visible image for the human eye) is 870 nm.

\subsection{Consideration 2}

2.2.1. Why don't you develop the IR emitting display? The authors confirmed above wavelength and phenomenon by placing an OHP sheet on which multiple numbers of tulip flowers were printed on the edge illuminated light guide panel, and photo recording the OHP film illuminated by the infrared light emitting LED (wavelength $870 \mathrm{~nm}$ ) from behind the light guide panel. The result of this experiment confirmed that the human eye did not recognize the IR light but the image sensor detected the IR light as a whitish light, and the quality of the image recorded was deteriorated (refer Figure 1).

The above experiment simulates the display method of images on the LCD display panel, and in a similar way, it is possible to impair the quality of the video recording by placing IR light sources behind the LCD panel. However, in order to introduce the IR emitting display, replacement of the existing display is necessary, which involves significant costs as well as troublesome transportation, replacement, and installation.

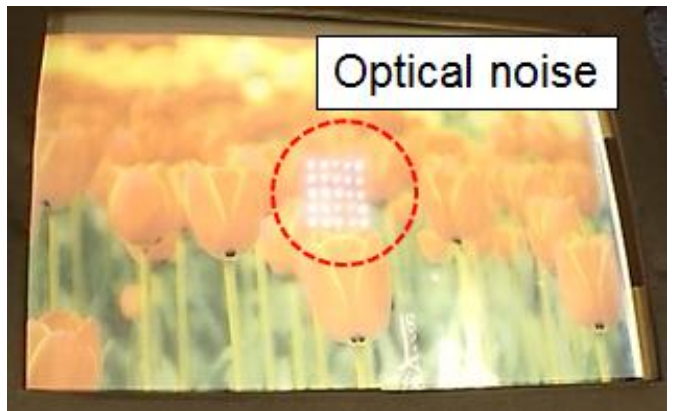

Figure 1. A photographic image illuminated with 25 infrared light emitting LEDs located behind the edge illuminated light guide plate.

\subsubsection{Why not use an IR emitting panel using the} transparent light guide panel and the IR light? The authors built a transparent panel that emits IR light as a test piece inspired by the transparent protection panel of a display screen. This is based on the consideration that the existing display can be used continuously without replacement and on the consideration that such panel is more light weight and thinner compared with a box-type IR light emitting device, which is installed in front of the display screen [7].

The authors installed the acrylic light guide panel that incorporates 40 infrared light emitting LEDs (wavelength $870 \mathrm{~nm}$ ) on the upper and lower edges in front of the display screen. With an image displayed on the screen and the light guide illuminated by the IR light emitting LEDs from both sides, photographs of the display screen were taken by a compact digital camera (Panasonic DMC-FX77). It was found that the recorded contents contained almost no optical noise (see Figure 2). This is because the amount of beads contained in the light guide to scatter light is small in the case of the transparent panel (with an increase in the amount of beads, the intensity of scattered light increases but the transparency of the panel diminishes)
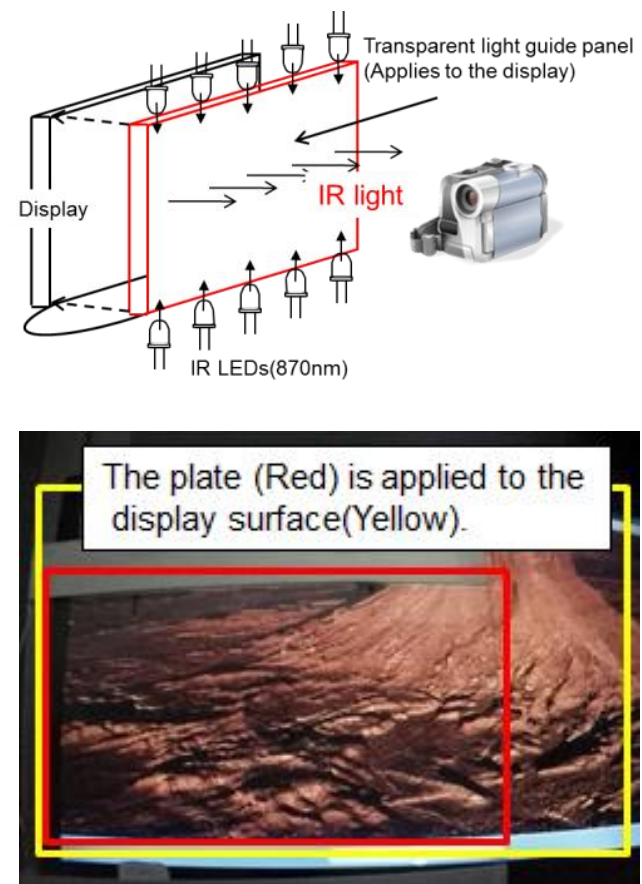

Figure 2. Conceptual diagram (above chart) and Result of experimentation.

\subsection{Consideration 3}

When the IR light emitting device is configured to allow installation on the existing display as described above, shooting of the onscreen contents is still possible if the device is removed by an insider. Accordingly, a system that detects removal of the IR light emitting device and activates appropriate protection of the content immediately after detection is required.

\subsection{Consideration 4}

The following facts were reported in Ref. [8] for photo shooting using an IR cut-off filter.

(1) An IR cut filter can be classified into two types: the type that absorbs IR light received on the filter (absorptive type) or the type that reflects IR light on the surface of the filter (reflective type). When such filter is installed in front of the camera lens, shooting of noiseless content becomes possible.

(2) The imaging element generally has high sensitivity in the IR range outside the visible 
range. Because of this, a filter with its IR light cut-off ratio adjusted according to the specifications of shooting of the digital camera is installed in front of the imaging element.

In order to verify the latter fact, the authors took photographs of the IR light emitting diodes (wavelength $870 \mathrm{~nm}$ ) using a compact digital camera (Panasonic DMC-FX77) and the single-lens reflex digital camera (Canon EOS Kiss X5) available from electronics retailers under illuminated and nonilluminated conditions. The results indicate that the compact camera detects IR light as visible light but the single-lens reflex camera does not (see Figure 3). This means that shooting of the content without noise is possible without installing an IR cut-off filter in front of the lens when a digital camera with higher specification is used.

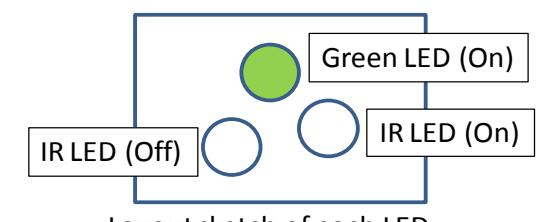

Layout sketch of each LED

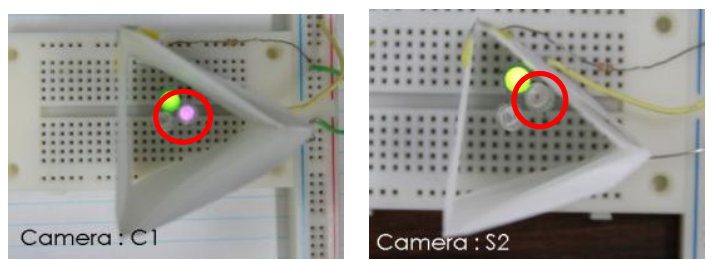

Figure 3. Obtained photographic image using digital compact camera (left) and digital single-lens reflex camera (right)

\subsection{Targets}

According to the considerations above, the authors determined the targets of the Countermeasures 1 and 2 as follows.

Countermeasure 1: The IR light emitting device should emit near infrared light at around $870 \mathrm{~nm}$ and should be flexible so that it can be attached on the existing display screen. If the IR light emitting device is removed, the content displayed should be appropriately protected.

Countermeasure 2: Detection of shooting of the screen should be possible. Use of either reflective type or absorptive type IR cut-off filter in $3 \mathrm{~cm}$ to 5 $\mathrm{cm}$ diameter installed in front of the camera lens or use of a higher specification camera should be considered as a method of such shooting.

\section{Development of Countermeasure 1}

\subsection{Development of the transparent sheet that emits IR light}

3.1.1. Study of the inorganic material Highly transparent glass phosphor that emits IR light (peak wavelength $1000 \mathrm{~nm}$ ) by accepting visible light (optical excitation) [9] had drawn the attention of the authors as a candidate material. The glass is produced by melting a mixture of powders from a small amount of rare earth oxides and supporting glass. Although rare earth oxides are used, they are relatively easily obtainable, and since the content is only a few percent of the supporting glass in weight, the material can be produced at relatively low cost.

Transparency and conversion efficiency of the glass phosphor are dependent on the types and content of rare earth oxides used, and the composition of the supporting glass. Because the glass phosphor, which the authors had introduced[3], showed yellow tint, a review of the composition of the glass was made, and removal of the tint and improvement of transparency were made successfully (see Figure 4). Observation of its emission spectrum indicated that emission of IR light has a peak at $870 \mathrm{~nm}$.

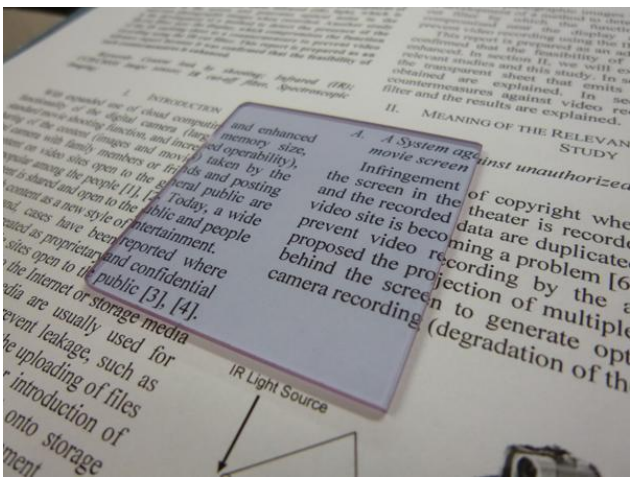

Figure 4. Glass phosphor with its transparency improved after review of the composition

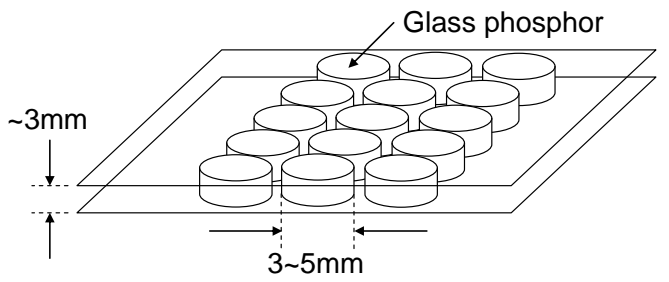

Figure 5. A concept of filling glass phosphor granules in the resin. Such a concept makes it possible to produce the prototype of a transparent flexible sheet.

The authors produced a prototype using transparent resin (silicon) filled with many small glass phosphor granules based on the concept shown in Figure 5, in order to produce a transparent flexible sheet that contains large amounts of glass phosphor. 


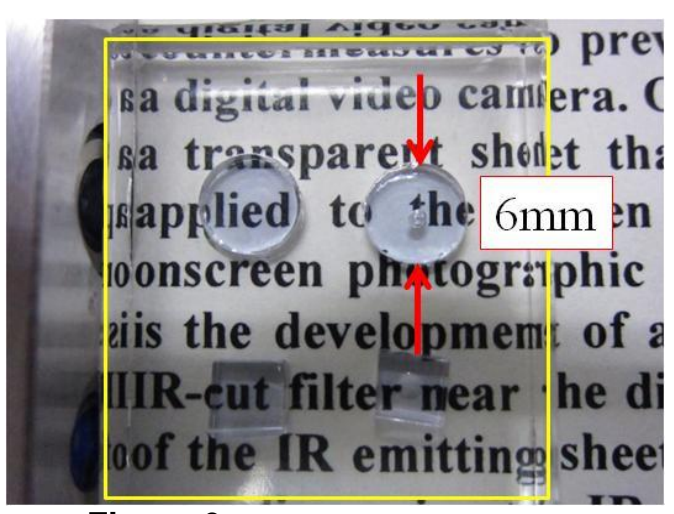

Figure 6. The prototype produced.
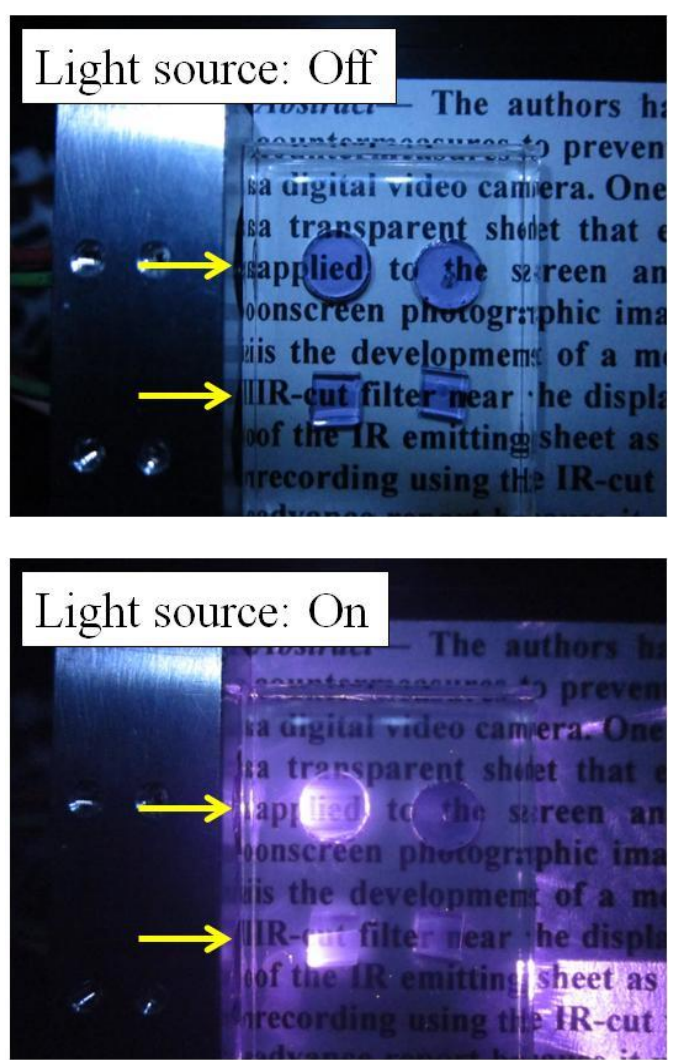

Figure 7. A photograph taken without illumination by visible light (above chart) and with illumination by visible light.

In the prototype produced (see Figure 6), the glass phosphor granules can be identified, but such phenomenon can be eliminated by equalizing the refractive index of both materials as reported by the authors in Ref. [3]. Next, the authors took photographs of the printed material using the compact digital camera with the prototype sheet placed on the printed material and illuminated by visible light from the side. The result indicated that letters on the print became hard to identify due to illumination by the IR light (see Figure 7).

3.1.2. Availability of the organic material By the way, we have also been paying close attention to the development trend of the organic EL (electroluminescent) screens. As shown in Figure 8, the Organic EL sheet is shin, transparent, highly flexible, and can emit visible or invisible light with lower electrical power than the inorganic material[10, 11]. Besides, we can put this sheet on the transparent display [12] or showcase to prevent shooting onscreen contents or displayed objects. In many cases, taking photos is prohibited in the gallery and museum.

At the current moment, it is not easy to popularize this organic EL sheet as a countermeasure 1 due to its high price. However, we are considering applying the organic EL sheet as a security sheet in the future.

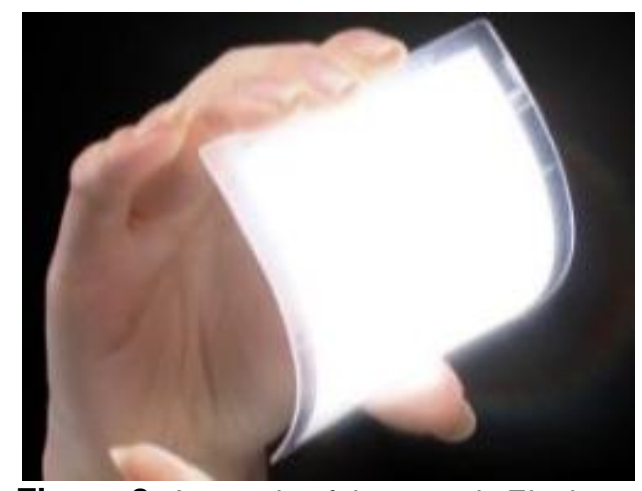

Figure 8. A sample of the organic EL sheet.

\subsection{Development of the system that detects removal of the sheet}

The authors developed a sensor that detects removal of the IR light emitting sheet and a system where protection of the content displayed on the screen is activated by the sensor detection.

Figure 9 shows the system outline. Sensors are installed on two opposing sides of the IR light emitting sheet, which are connected to the input terminal of the voltmeter. The output of the voltmeter and the PC are connected by a USB cable, and the PC and the display are connected by the display cable. The sensor does not require electrical power input but generates voltage when a bending force is applied. Because the sensors are installed on the two sides of the sheet, voltage is produced whenever a person tries to peel off the sheet in the direction of (a) or (b). The software that monitors output voltage of the voltmeter is installed on the PC, and when the voltage exceeds the threshold value, the software determines that the sheet is being peeled off and displays the warning screen (the warning screen prevents video recording of the content displayed on the screen). 


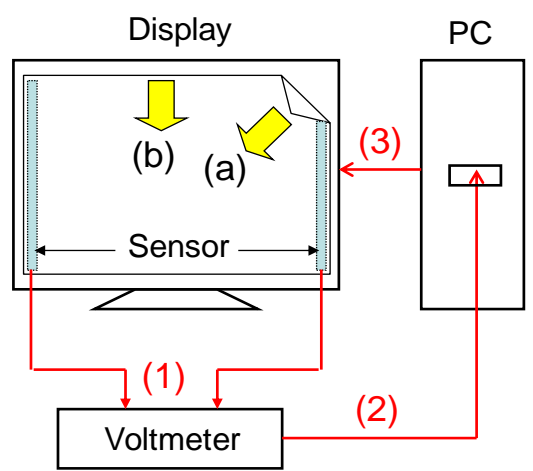

Figure 9. A system for detecting the removal of the sheet. When removal of the sheet is detected, the system displays the warning screen.

\subsection{Development of the system that detects cut of the sheet}

The authors developed a system that protects the content displayed on the screen from shooting by the interlock incorporating the sensor that detects cutting of the IR light emitting sheet using a cutter, assuming an act of shooting where the sheet is cut by a cutter and is peeled off partly or entirely to expose the screen (see Figure 10).

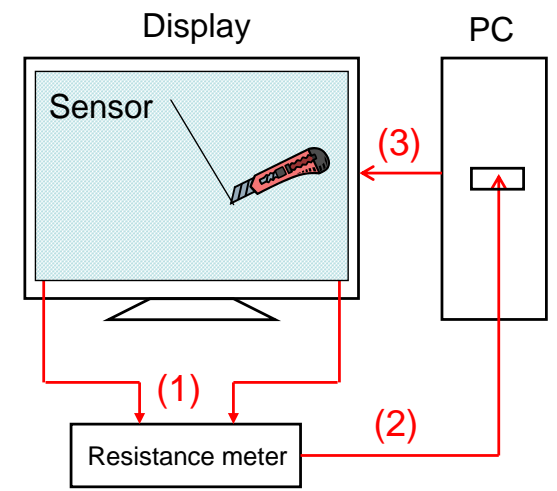

Figure 10. A system for detecting the cut of the sheet. When cut of the sheet is detected, the system displays the warning screen.

Very thin conductive wire is embedded (in unicursal repetitive pattern) into the entire half-sheet, and both ends of the wire (+ and - poles) are connected to an ohmmeter (transparency of the sheet can be maintained more than $80 \%$ as far as width of the wire is in micrometer order). The output of the resistance meter and the PC are connected by a USB cable, and the PC and the display are connected by the display cable. The software that monitors output resistance of the resistance meter is installed on the $\mathrm{PC}$, and when the resistance exceeds the threshold value (i.e., a cut more than about $2 \mathrm{~cm}$ in length is detected), the software determines that the sheet is being cut and displays the warning screen (the warning screen prevents video recording of the content displayed on the screen).

\section{Development of countermeasure 2}

In this section, the authors explain two approaches for detection of photo shooting of the display screen.

\subsection{Approach 1}

A nondestructive test in which the state of the object for inspection was photographed with near IR light irradiated on the object is known [10]. The authors decided to use this method to detect the presence of the shooting equipment (IR cut filter or the camera). Figure 11 shows the conceptual diagram. A near IR light source and a camera that has multiple imaging areas are installed on the display, and spectroscopic images are taken at an interval of a few seconds. Software that processes spectrographic images is installed on the PC, which determines possible presence of the shooting equipment. When presence of the shooting equipment is detected, the software makes a window to protect the content displayed on the screen or turns off the display.

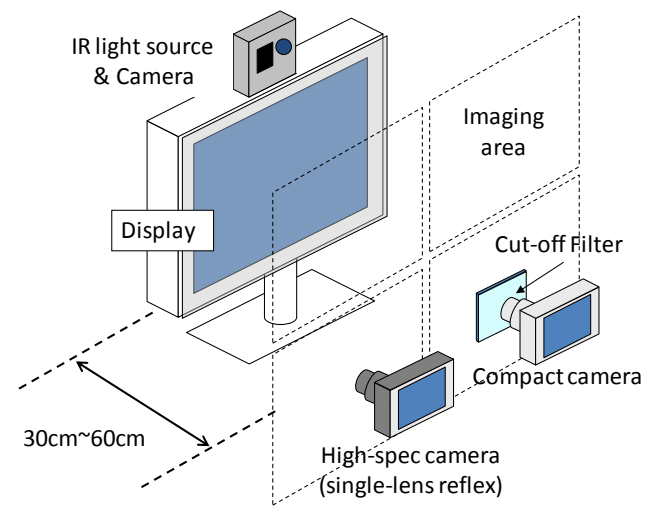

Figure 11. The system monitors multiple imaging areas and detects the presence of the shooting equipment (the IR cut filter or the camera). In this experiment, six IR emitting LEDs arranged in ring pattern are used as the light source.

The authors developed the following decision logic based on the characteristics of the spectrographic image reported in Ref. [3] and the characteristic of the spectrographic image newly taken (see Figure 12).

(1) When a black circle with a diameter of $3-5 \mathrm{~cm}$ is included in the spectrographic image, the decision is made that an absorptive type IR cutoff filter on which surface anti-reflective coating is applied is likely to present in front of the display, and protection of the content is activated.

(2) When several bright spots are included in the spectrographic image, the decision is made that a camera is present in front of the display, and protection of the content is activated. This phenomenon is due to reflection of the IR light irradiated by the following objects. 
- Reflective type IR light cut-off filter

- Absorptive type IR light cut-off filter without anti-reflective coating (a cut-off filter without such coating reflects about $4 \%$ of light incident on its surface like conventional glass)

- Lens of the camera

- Metal or plastic material (used as a body of the camera)

When this decision logic is used, detection of shooting with the IR cut-off filter installed in front of the lens of the camera or shooting by the high specification camera without the IR cutoff filter is possible. However, the criteria for this decision logic are not precise, and the possibility remains that protection of the content may be erroneously activated when glass, metallic, and/or plastic material in a diameter of $3-5 \mathrm{~cm}$ not of a camera is present in front of the display. Accordingly, the authors are trying to improve the accuracy of the decision in combination with the other image processing techniques.
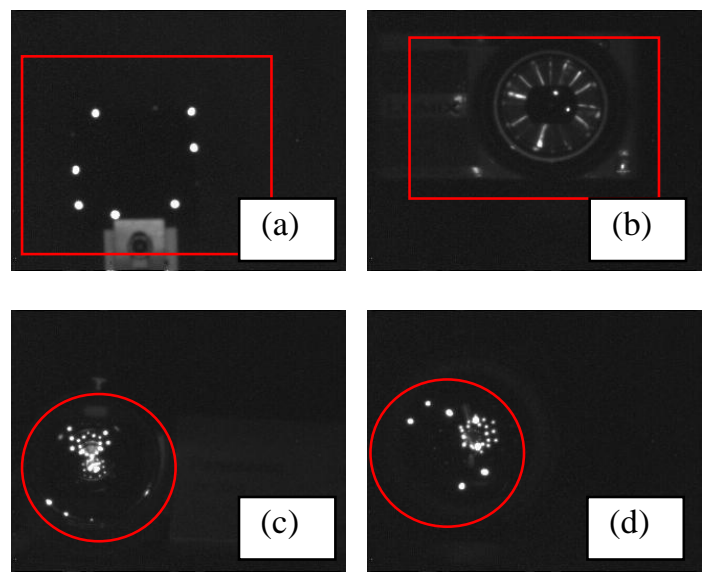

Figure 12. Spectrographic images (a: IR cut-off filter without anti-reflective coaling, b: Body of the compact camera, c: Lens of the video camera, $d$ : Lens of the single-lens reflex camera)

\subsection{Approach 2}

We have been trying to develop the detection system of shooting using Microsoft's Kinect-for Windows system, where detection of shooting holding a camera by both hands, a single hand, or on a tripod (i.e., posture of photo shooting) is possible. The Kinect system employs a motion detection sensor incorporating an RGB camera and a distance sensor, which captures the position of a human body and its motion. We have been developing software that enables real-time detection of shooting in the situation as shown in Figure 13 by tracking movement of the arms of a person facing the display or by identifying an article that remains stationary for certain period of time.

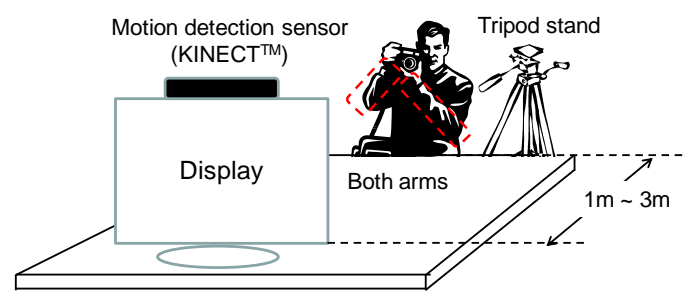

Figure 13. Detection of shooting using Kinect(TM).

Kinect-for-Windows has 2 distance measurement mode: One is Normal mode (measureable range: approx. $1 \mathrm{~m}$ to $3 \mathrm{~m}$ from Kinect), the other is Near mode (measureable range: approx. $40 \mathrm{~cm}$ to $1 \mathrm{~m}$ from Kinect). We developed the software which fitted for each mode.

4.2.1. Normal mode: This mode and software are suitable for the person who is standing/setting tripod and taking photo of onscreen contents. We confirmed that detection of a person aiming a camera at the display held by both or a single hand in front of the display, and a camera held on a tripod is possible (see Figure 14).
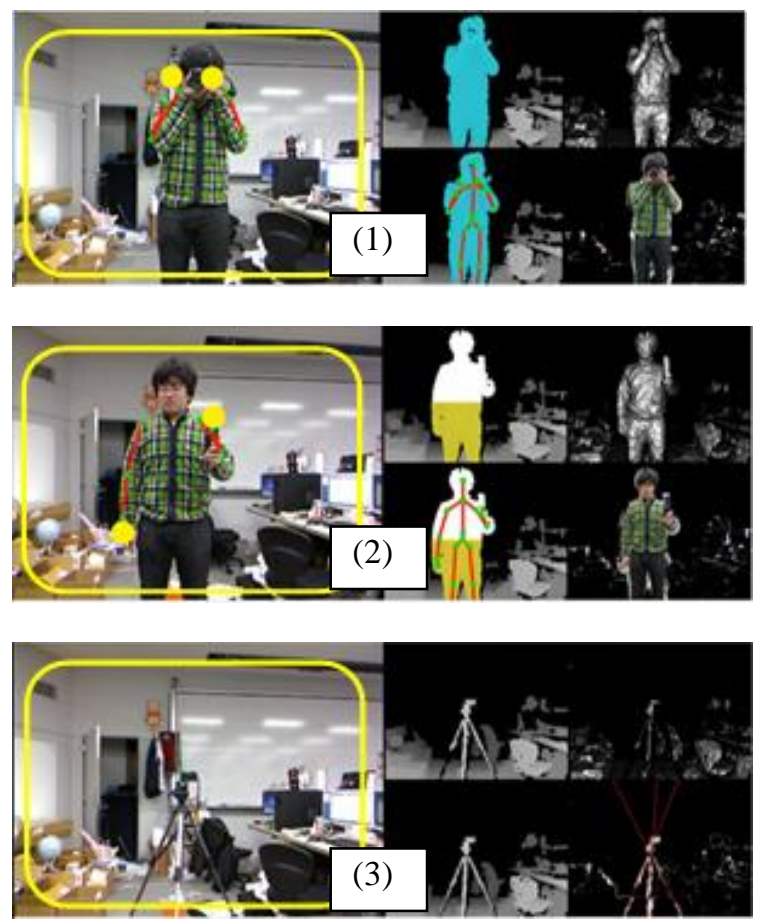

Figure 14. Detection result images (1: aiming a camera held by both hands, 2 : aiming a camera held by a single hand, 3 : presence of a tripod)

4.2.2. Near mode: This mode and software are suitable for the person who is taking photo of the onscreen contents during his/her desk work. We also confirmed that detection of a person aiming a camera at the display held by both or a single hand in front of the display, and a camera held on a tripod is possible (see Figure 15). 

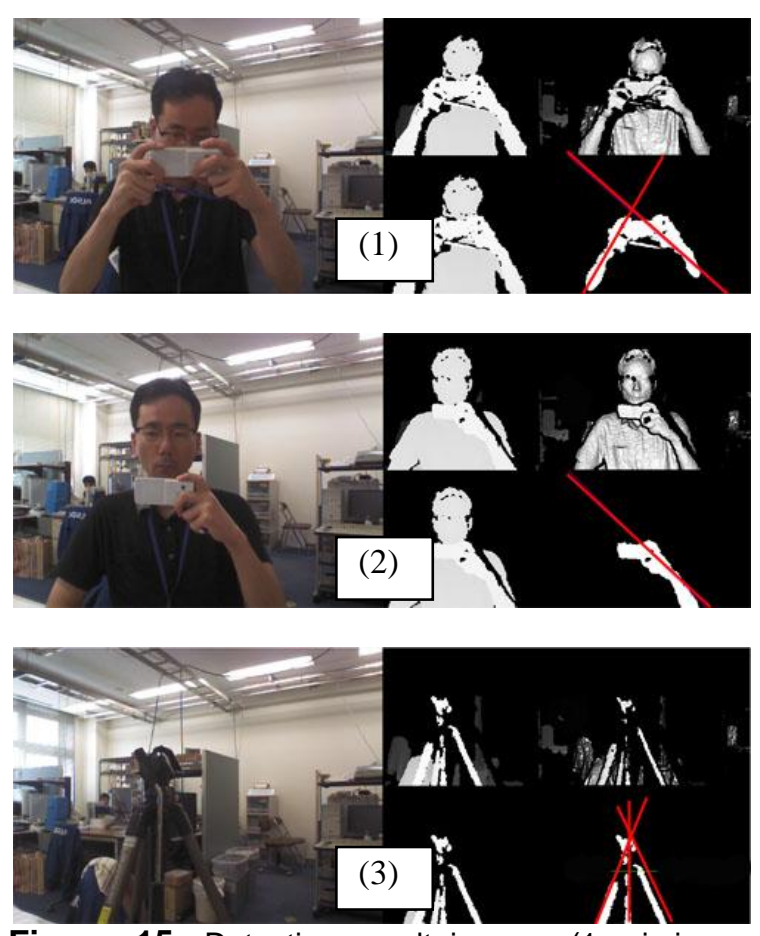

Figure 15. Detection result images (1: aiming a camera held by both hands, 2: aiming a camera held by a single hand, 3: presence of a tripod)

\section{Conclusion}

In this report, we proposed two countermeasures against content leaks from photo shooting, and presented the results of such countermeasures obtained by the experiment of 2 approaches. We intend to continue the research and development of the following items in order to enhance the feasibility of the respective countermeasures.

Countermeasure 1:

(1) Production and evaluation of the prototype IR light emitting sheet after production of the glass phosphor with a high level of transparency and conversion efficiency that has a refractive index equal to that of the resin.

(2) Development of the thin, transparent and flexible organic EL (electroluminescence) sheet which emits near-infrared light.

Countermeasure 2: Enhancement of accuracy in detection of recording in combination with different types of image processing technologies and by conducting experiments in a variety of situations.

\section{References}

[1] CNN World, (2011) 'Japanese coast guard member admits to leaking collision video', http:// articles. cnn. com/ 2010-11-10/ world/ japan. china. boat. video_1_crash-video-coast-guard-chinese-fishing?_s =PM:WORLD (11 December 2011).
[2] IBNLive.com India, (2011) 'Sudhir Mishra's unreleased film leaked on YouTube', http://ibnlive.in.com/news/sudhir-mishras-unreleased-filmleaked-on-youtube/108901-8.html (11 December 2011).

[3] Fujikawa, M., Akimoto, J., Oda, F., Moriyasu, K., Fuchi, S., Takeda, Y., (2011) 'Study of countermeasures for content leaks by video recording', Proc. of the Sixth International Conference on Availability, Reliability and Security (ARES).

[4] The New York Times, (2011) 'Japan Investigates Online Posting of Obama Flight Plans', http:// www.nytimes.com/2011/09/11/world/asia/11japan.html (11 December 2011).

[5] wordiQ.com, (2011) 'Copyright violation - Definition', http://www.wordiq.com/definition/Copyright_violation (11 December 2011).

[6] Yamada, T., Gohshi, S., Echizen, I., (2010) 'Reshooting prevention based on difference between sensory perceptions of humans and devices', Proc. of the IEEE International Conference on Image Processing (ICIP)

[7] Yamada, T., Gohshi, S., Echizen, I., (2011) 'Method for preventing illegal recording of displayed content based on differences in sensory perception between humans and devices', Proc. of the Computer Security Symposium 2011 (CSS), Japanese text.

[8] Yamada, T., Gohshi, S., Echizen, I., (2011) 'Countermeasure of reshooting prevention against attack with infrared-cut filter', Proc.of the IPSJ Symposium on Computer Security (CSS) 2010, Japanese text.

[9] Fuchi, S., Sakano, A., Takeda, Y., (2008) 'Wideband infrared emission from $\mathrm{Yb} 3+-$ and $\mathrm{Nd} 3+-$ doped Bi2O3B2O3 glass phosphor for an optical coherence tomography light source', Japanese Journal of Applied Physics, pp.7932-7935.

[10] M. Noda, N. Kobayashi, M. Katsuhara, A. Yumoto, S. Ushikura, R. Yasuda, N. Hirai, G. Yukawa, I. Yagi, K. Nomoto, and T. Urabe, (2010) 'A Rollable AM-OLED Display Driven by OTFTs’, SID 10 Digest 710.

[11] Arizona State University, 'The Flexible Display Center', http://flexdisplay.asu.edu/ (31 August 2012)

[12] C. Zibreg, 'iPhone 5 with transparent iClear Retina display and laser projection keyboard', http://www.idownloadblog.com/2012/06/15/iphone-5transparent-mockup/ (31 August 2012)

[13] Driver, D., Didona, K., (2011) 'On-line High-Speed NIR Diffuse-Reflectance Imaging Spectroscopy In Food Quality Monitoring', http://www.headwallphotonics.com/downloads/SPIE-NIRImaging.pdf (11 December 2011). 